\title{
Effect of Ge-doping on the short-wave, mid- and far- infrared intersubband transitions in GaN/AIGaN heterostructures
}

\author{
Caroline B Lim ${ }^{1}$ Akhil Ajay ${ }^{1}$, Jonas Lähnemann², Catherine Bougerol ${ }^{3}$ and \\ Eva Monroy ${ }^{1}$ \\ ${ }^{1}$ University Grenoble-Alpes, CEA, INAC, PHELIQS, 17 av. des Martyrs, 38000 Grenoble, \\ France \\ ${ }^{2}$ Paul-Drude-Institut für Festkörperelektronik, Hausvogteiplatz 5-7, 10117 Berlin, Germany \\ ${ }^{3}$ University Grenoble-Alpes, CNRS, Institut Néel, 25 av. des Martyrs, 38000 Grenoble, \\ France
}

\section{Abstract}

This paper assesses the effects of Ge-doping on the structural and optical (band-to-band and intersubband) properties of GaN/AlGaN multi-quantum wells designed to display intersubband absorption in the short-wave, mid- and far-infrared ranges (SWIR, MIR, and FIR, respectively). The standard $c$-plane crystallographic orientation is considered for wells absorbing in the SWIR and MIR spectral regions, whereas the FIR structures are grown along the nonpolar $m$-axis. In all cases, we compare the characteristics of Ge-doped and $\mathrm{Si}$ doped samples with the same design and various doping levels. The use of Ge appears to improve the mosaicity of the highly lattice-mismatched GaN/AIN heterostructures. However, when reducing the lattice mismatch, the mosaicity is rather determined by the substrate and does not show any dependence on the dopant nature or concentration. From the optical point of view, by increasing the dopant density, we observe a blueshift of the photoluminescence in polar samples due to the screening of the internal electric field by free carriers. In the intersubband absorption, on the other hand, there is a systematic improvement of the linewidth when using Ge as a dopant for high doping levels, whatever the spectral region 
under consideration (i.e. different quantum well size, barrier composition and crystallographic orientation).

Keywords: GaN, AlGaN, quantum well, intersubband, nonpolar, doping, Ge

\section{Introduction}

GaN/AlGaN nanostructures have shown their potential for the development of new intersubband (ISB) optoelectronic devices, with the possibility to cover almost the whole infrared (IR) spectrum [1,2]. Their large conduction band offsets and sub-picosecond ISB relaxation times support the development of ultrafast photonic devices operating at telecommunication wavelengths [3-5]. Additionally, the large energy of the longitudinaloptical phonon in $\mathrm{GaN}(92 \mathrm{meV}, 13 \mu \mathrm{m}$ ) opens prospects for $\mathrm{THz}$ quantum cascade lasers operating at room-temperature [6,7]. GaN is transparent for most of the IR spectral region, namely for wavelengths longer than $360 \mathrm{~nm}$, except for the Reststrahlen band (phonon absorption band) located between 9.6 and $19 \mu \mathrm{m}$. Using $c$-plane GaN/AlN quantum wells (QWs), the ISB absorption can be tuned in the 1.0-3.5 $\mu \mathrm{m}$ wavelength range by changing the QW thickness from $1 \mathrm{~nm}$ to $7 \mathrm{~nm}$ [8-12]. To shift the absorption towards longer wavelengths, it is necessary to reduce the polarization-induced internal electric field in the QWs, which can be attained by using ternary AlGaN barriers with reduced $\mathrm{Al}$ mole fraction. Varying the geometry and composition of the barriers, the ISB absorption can be tailored to cover the range up to $10 \mu \mathrm{m}$ [13-18]. Reducing the ISB transition energy below $60 \mathrm{meV}$ (wavelength $>20 \mu \mathrm{m}$ ) requires further band engineering to compensate the internal electric field in the QWs, which is only achieved by implementation of complex multi-layer QW designs [19-22]. The use of nonpolar crystallographic orientations, particularly the $m$-plane, 
is a promising alternative to obtain $\mathrm{GaN} / \mathrm{AlGaN}$ QWs without internal electric field [23-25].

The development of ISB devices requires a fine tuning of the n-type doping density, particularly in photodetectors and switches, where the first quantum-confined energy level of the conduction band has to be populated to allow electron transitions to higher levels. To measure ISB transitions in GaN/AIN QWs, large n-type doping densities $\left(N_{\mathrm{D}}>1 \times 10^{18} \mathrm{~cm}^{-3}\right)$ are required. The charge density has a significant impact on the transition energy and linewidth due to many-body effects such as depolarization shift and exchange interaction, as well as scattering by ionized impurities [8,23,26-29]. Furthermore, such doping concentrations can introduce strain, eventually leading to structural defects [30] or roughness at the heterointerfaces [31], which constitutes an additional source of carrier scattering. In general, studies of ISB transitions in III-nitrides have been performed using Si as n-type dopant, even though it is known that it introduces tensile strain which can lead to a structural degradation [30]. Ge is considered as an alternative n-type dopant, with improved performance in comparison to Si for doping levels in excess of $10^{19} \mathrm{~cm}^{-3}$ [32-35]. Thanks to its ionic radius and metal-nitrogen bond length similar to that of $\mathrm{Ga}$, the $\mathrm{Ge}$ occupancy of a Ga lattice site is expected to induce far less lattice distortions compared with Si.

In this paper, we assess the effect of Ge doping on the structural quality, band-to-band and ISB properties of polar $c$-plane GaN/AlGaN multi-quantum-wells (MQWs) designed for ISB absorption in the short-wave (SWIR, 1.4-3 $\mu \mathrm{m}$ ) and mid-infrared (MIR, 3-5 $\mu \mathrm{m}$ ) ranges, and of nonpolar $m$-plane GaN/AlGaN MQWs, designed for ISB absorption in the farinfrared (FIR, $>20 \mu \mathrm{m})$ domain. We systematically compare identical structures doped with Ge and Si.

\section{Methods}


The band structure and quantum-confined electron levels were modeled using the Nextnano3 $8 \times 8$ k.p self-consistent Schrödinger-Poisson solver [36] with the material parameters described in ref. [37]. The structure was treated in one dimension. The simulation covered a section of the MQW superlattice including three QWs, with periodic boundary conditions. With this simulation cell, we can confirm that the periodicity of the structure is correctly treated by checking that the results are identical in the three QWs.

The MQW structures were synthesized by plasma-assisted molecular-beam epitaxy (PAMBE). Thanks to its low growth temperature, this technique is particularly adequate to the fabrication of heterostructures with chemically abrupt interfaces [38]. Furthermore, it offers compatible growth conditions for the $c$-plane and the $m$-plane of $\mathrm{GaN}$ [39], and $\mathrm{Si}$ and Ge can be incorporated as dopants during the growth process, without perturbation of the growth kinetics $[35,40]$. For $c$-plane samples, the substrates were either $1-\mu m$-thick AlN-onsapphire templates with a dislocation density $\approx 10^{9} \mathrm{~cm}^{-2}$ (GaN/AlN MQW structures) or 4$\mu$ m-thick GaN-on-Si(111) templates with a dislocation density $<5 \times 10^{9} \mathrm{~cm}^{-2}(\mathrm{GaN} / \mathrm{AlGaN}$ MQW structures), both deposited by metalorganic vapor phase epitaxy. For $m$-plane samples, the substrates were $5 \mathrm{~mm} \times 20 \mathrm{~mm}$ semi-insulating $m$-GaN platelets sliced from (0001)-oriented GaN boules synthesized by hydride vapor phase epitaxy (resistivity $>10^{6} \Omega \mathrm{cm}$, dislocation density $<5 \times 10^{6} \mathrm{~cm}^{-2}$ ). In all cases, the growth was performed under slightly Ga-rich conditions [24,25,39,41], at a substrate temperature of $720^{\circ} \mathrm{C}$, and with a nitrogen-limited growth rate of $0.5 \mathrm{ML} / \mathrm{s}(\approx 450 \mathrm{~nm} / \mathrm{h})$. A 200 -nm-thick, non-intentionally-doped GaN buffer layer was deposited prior to the MQW, which was capped with $30-50 \mathrm{~nm}$ of $\mathrm{AlGaN}$ using the same $\mathrm{Al}$ content as for the barriers. The $\mathrm{GaN}$ wells were doped with either Si or Ge, at concentrations in the $5 \times 10^{11} \mathrm{~cm}^{-2}$ to $6 \times 10^{13} \mathrm{~cm}^{-2}$ range, as indicated in the list of samples in Table 1. The doping level was calibrated by Hall effect measurements in reference $c$-plane $\mathrm{GaN}: \mathrm{Si}$ and $\mathrm{GaN}: \mathrm{Ge}$ layers grown on AlN-on- 
sapphire templates.

The surface morphology of the layers was studied by atomic force microscopy (AFM) in the tapping mode using a Dimension 3100 system. The periodicity and structural properties of the MQWs were measured by high-resolution x-ray diffraction (HR-XRD) using a Rigaku SmartLab x-ray diffractometer with a 4-bounce Ge(220) monochromator and a 0.114 degree collimator in front of the detector. Samples were analyzed by high-angle annular dark-field scanning transmission electron microscopy (HAADF-STEM) performed in an FEI Titan Themis microscope operated at $200 \mathrm{kV}$. Cathodoluminescence (CL) measurements at $10 \mathrm{~K}$ were performed in a Zeiss Ultra-55 field-emission scanning electron microscope fitted with a Gatan MonoCL4 system, using an acceleration voltage of $3 \mathrm{kV}$ and a current of about $400 \mathrm{pA}$. The emission from the sample was collected by a parabolic mirror and guided into a grating monochromator equipped with a liquid-nitrogen-cooled charge-coupled device (CCD) camera. Spectral line-scans were recorded with an integration time of $1 \mathrm{~s}$ per spectrum. Photoluminescence (PL) spectra were obtained by excitation with a continuouswave solid-state laser $(\lambda=244 \mathrm{~nm})$, with an excitation power around $100 \mu \mathrm{W}$ focused on a spot with a diameter around $100 \mu \mathrm{m}$. The emission from the sample was collected by a Jobin Yvon HR460 monochromator equipped with an ultraviolet-enhanced CCD camera. To assess the ISB properties of the MQWs, transmission measurements employing Fourier transform infrared (FTIR) spectroscopy were performed using a Bruker V70v spectrometer. For characterization in the SWIR and MIR ranges, the FTIR spectrometer was equipped with a halogen lamp and a nitrogen-cooled mercury-cadmium-telluride detector. All samples were polished at $45^{\circ}$ (sapphire and $m$-GaN substrates) or at $30^{\circ}$ ( $\mathrm{Si}$ substrates) to form multipass waveguides allowing 4-5 interactions with the active region. For characterization in the FIR, we used a mercury lamp, a Si beam splitter and a helium-cooled Si bolometer. In the latter case, two pieces of each sample were placed face-to-face on the cold finger of a helium- 
cooled cryostat. All the samples were tested in transmission mode using a polarizer adapted to the targeted infrared region to discern between the transverse-electric (TE) and transversemagnetic (TM) polarized light.

\section{Experimental results}

\section{A. SWIR absorption in c-plane GaN/AIN MQWs}

We have studied a series of structures containing 25 periods of GaN/AlN $(1.8 \mathrm{~nm} / 3 \mathrm{~nm})$ MQWs designed to show ISB absorption at $0.729 \mathrm{eV}(1.70 \mu \mathrm{m})$ for low doping levels (samples S11 to S16 in Table 1). Figure 1 shows a sketch of the structure and the conduction band profile of one of the QWs, including the location of the first and second quantumconfined electron levels and their associated squared wavefunctions. Note that the potential profile of the MQW displays the triangular pattern characteristic of GaN/AlN heterostructures, due to the internal electric field associated to the difference in spontaneous and piezoelectric polarization between the wells and the barriers. The electric field results in a redshift of the band-to-band transitions (quantum confined Stark effect) and a blueshift of the ISB transitions with respect to square MQWs [24]. Additionally, the fact that the gravity centers of the wavefunctions associated to the first hole level and the first and second electron confined levels are shifted along the growth axis leads to a decrease of the dipole matrix elements associated to both of the band-to-band and ISB transitions. Growth along nonpolar crystallographic orientations, such as the $<1-100>m$ axis, inhibits the appearance of internal electric field, with the resulting enhancement of the dipole matrix elements. However, the lattice mismatch in the nonpolar planes is larger, which leads to a high density of dislocations, cracks and stacking faults when the average $\mathrm{Al}$ concentration in the structure exceeds $\approx 10 \%$ [42]. Therefore, in architectures for ISB optoelectronics, the use of nonpolar 
crystallographic orientations is restricted to applications in the FIR.

After growth, the periodicity of the structure was confirmed by measuring $\omega-2 \theta$ scans of the (0002) x-ray reflection. Typical diffractograms for Ge-doped and Si-doped structures are presented in Fig. 1(c), together with a simulation performed with the Rigaku SmartLab Studio software. The simulation provides a good reproduction of the experimental result assuming that the MQW presents the in-plane lattice parameters of an AlGaN layer with the average $\mathrm{Al}$ concentration of the MQW, i.e. the MQW structure is fully relaxed. This is expected to be the case in view of the thickness of the MQW, following the study in ref. [43]. The values of the MQW period extracted from the intersatellite angular distance in the diffraction pattern are summarized in Table 1. Also, the full width at half maximum (FWHM) of the rocking curves was measured for the AlN substrate and the MQW zeroorder reflection [MQW 0 peak in Fig. 1(c)], obtaining an average FWHM of $0.19 \pm 0.01^{\circ}$ for Si-doped MQWs and $0.16 \pm 0.01^{\circ}$ for Ge-doped MQWs, to be compared with $0.057 \pm 0.006^{\circ}$ for the AlN-on-sapphire template. The broadening of the MQW rocking curve in comparison with the substrate is due to strain relaxation. On the other hand, Gedoped MQWs systematically present $\approx 18 \%$ narrower rocking curves than their Si-doped counterparts for all the doping levels. This correlation indicates that, for similar substrate quality, Ge-doping results in structures with better mosaicity than when using Si-doping.

To assess the band-to-band properties of the MQWs, the PL spectra of all the samples were measured at $5 \mathrm{~K}$. The results in terms of peak emission energy are shown in Table 1, and the spectra are displayed in Figs. 2(a) and (b). Both for Ge- and Si-doping, the spectra present a multipeak structure resulting from monolayer fluctuations of the well thickness [8]. Increasing the dopant density induces a blueshift of the PL energy, which is assigned to the screening of the internal electric field by the free carriers [44], as well as a broadening of the emission peak, which is due to the Burstein-Moss effect [45]. The total shift in emission 
energy and the energy broadening are similar for both dopants. Calculated values of the band-to-band transition energy obtained using the nextnano3 software are indicated by black triangles in the figure. These calculations, which take into account the sample period and the screening of the internal electric fields by free carriers, provide a reasonable fit to the experimental results. The agreement confirms the incorporation and activation of both $\mathrm{Si}$ and Ge in the QWs.

The vertical homogeneity of the MQW stack has been studied by low-temperature $(10 \mathrm{~K}) \mathrm{CL}$, recording a spectral line-scan on a cross-section of sample S16 cleaved along the growth direction. Figure 3 displays the obtained spectral map, along with a sketch of the sample. Following the growth axis, the MQW emission broadens, redshifts and gains intensity, which is explained by the gradual relaxation of misfit strain induced by the AlN substrate, which can extend over 10-20 MQW periods [43]. Theoretical calculations predict a redshift of $11 \mathrm{~nm}$ when the superlattice evolves from being fully strained on AlN to fully relaxed.

To assess the ISB properties of the samples, the absorption in the SWIR range was measured at room temperature by FTIR spectroscopy. Figures 2(c) and (d) show the normalized absorption spectra for TM-polarized light. As explained in the PL case, the multipeak structure of the spectra is due to monolayer thickness fluctuations in the wells [8]. The ISB absorption peak energies for the various samples are presented in Table 1, together with the magnitude of the absorption per pass. The value of absorption should be taken cautiously, since the accumulated error associated to the calculation of the waveguide length and incident angle can reach $\pm 20 \%$. However, comparing S11-12 and S13-14, we can conclude the magnitude of the absorption scales linearly with the doping density, as expected, and a saturation is observed for S15-16, which is explained by the Fermi level approaching the excited state $\mathrm{e}_{2}$ in these heavily doped structures. As expected, the 
absorption resonances are blueshifted when increasing the doping concentration, which is due to many-body effects, namely exchange interaction and depolarization shift. The magnitude of these effects has been quantified as described in ref. [46], giving the theoretical transition energies indicated by black triangles in Fig. 2. In general, the magnitude of the spectral shift agrees well with the theoretical expectations for both $\mathrm{Si}$ and Ge. Regarding the absorption linewidth, we observe that Si-doping leads to stronger broadening of the absorption peak, as summarized in Fig. 2(e). Thus, in this case, broadening is not dominated by the scattering by ionized impurities or electron-electron interaction, but rather by interface roughness.

\section{B. MIR absorption in c-plane GaN/AIGaN MQWs}

To extend the study to the MIR range, the AlN barriers were replaced by AlGaN, and the width of the QWs was increased. Therefore, this study focuses on structures consisting of 30 periods of $\mathrm{GaN} / \mathrm{Al}_{0.33} \mathrm{Ga}_{0.67} \mathrm{~N}(4 \mathrm{~nm} / 3 \mathrm{~nm}) \mathrm{MQWs}$, designed to show ISB absorption at $240 \mathrm{meV}(5.2 \mu \mathrm{m})$ at low doping levels (samples S21 to S26 in Table 1). A 2-nm-thick region at the center of the GaN QWs was homogeneously doped with $\mathrm{Si}$ or Ge, to reach the surface dopant densities described in Table 1. Figure 4 shows a sketch of the structure as well as the conduction band diagram of one of the QWs in the middle of the stack. Typical HR-XRD $\omega-2 \theta$ scans of the (0002) reflection for these samples are shown in Fig. 4(c). The diffractograms are complex due to the presence of an additional multiple-heterostructure close to the silicon/GaN interface with a period of $\approx 20 \mathrm{~nm}$. The purpose of this structure is to maintain the GaN under compressive strain during the cool down process after growth, thus preventing crack propagation. The simulation presented in the figure does not take into account the buffer layer, and assumes that the MQW presents the in-plane lattice parameters of an $\mathrm{AlGaN}$ layer with the average $\mathrm{Al}$ concentration of the MQW, as it is expected [47]. By comparison of the experimental diffractograms with the simulation, it is possible to identify 
the satellites corresponding to the PAMBE-grown GaN/AIN MQW structure and determine its period. The results are summarized in Table 1. Additionally, the structural quality was evaluated by measuring the FWHM of the rocking curves for the GaN template and the MQW zero-order reflection. The resulting data, also listed in Table 1, yields average values of $0.189 \pm 0.005^{\circ}$ and $0.187 \pm 0.005^{\circ}$ for Si-doped and Ge-doped MQWs, respectively, to be compared with $0.208 \pm 0.005^{\circ}$ for the GaN-on-Si(111) templates. We can hence conclude that the mosaicity of the samples shows no clear dependence on either the dopant nature or the doping density, and is rather determined by the substrate.

Concerning the band-to-band properties of the MQWs, the low temperature (5 K) PL spectra from samples with different doping levels of Ge and Si are presented in Figs. 5(a) and (b), respectively. Increasing the Ge-doping density results in a slight blueshift of the PL energy, as well as an asymmetric widening of the PL peak towards the low-energy side. The magnitude of the spectral shift, associated with the screening of the internal electric field, is significantly smaller than in the samples S11-S16 described in the previous section, in spite of the fact that the QWs are larger (4 $\mathrm{nm}$ in S21-S26 to be compared with $2 \mathrm{~nm}$ in S11-S16). This reduced shift is mainly due to the reduction of the internal electric fields when using $\mathrm{Al}_{0.33} \mathrm{Ga}_{0.67} \mathrm{~N}$ barriers instead of AlN. Looking at the PL spectra in Figs. 5(a-b), the emission from S25 appears surprisingly narrow for a sample with such a high doping level. A closer look at the spectrum reveals that the main emission consists of two peaks: a dominant "narrow" line at $3.46 \mathrm{eV}$ and a broader emission at higher energies, around $3.51 \mathrm{eV}$, with similar characteristics (in logarithmic scale, not shown) than the emission from S26. Comparing the two samples, the emission at $3.46 \mathrm{eV}$ in $\mathrm{S} 25$ is probably due to localized emission at structural inhomogeneities, but we did not manage to identify such defects in transmission electron microscopy images.

To assess the homogeneity of the MQW stack along the growth axis, a spectral line-scan 
of the low-temperature $(10 \mathrm{~K}) \mathrm{CL}$ was recorded on a cross-section of the sample with the highest Ge doping level (S26) cleaved along the growth direction (Fig. 6). In agreement with the PL experiments in Fig. 5(a), the emission from the MQW, peaking at $355 \mathrm{~nm}(3.49 \mathrm{eV})$, is strongly asymmetric. Its intensity increases along the growth axis, but the spectral shift is reduced in comparison with the GaN/AlN MQWs (see Fig. 3), which is consistent with the smaller lattice mismatch with the substrate. The luminescence from the AlGaN capping layer is visible as an extra emission peak centered around $327 \mathrm{~nm}(3.79 \mathrm{eV})$. There is no detectable emission from the $\mathrm{AlGaN}$ barriers, which points to a good charge transfer from the barriers to the wells.

The ISB properties of the MQWs were measured by FTIR at room temperature. As summarized in Table 1 and illustrated in Figs. 5(c) and (d), all samples present a TMpolarized absorption peak, which blueshifts for increasing doping levels. As in the SWIR set, the absorption scales sublinearly with the doping density, which is justified by the high doping levels that bring the Fermi level close to $\mathrm{e}_{2}$, or even beyond. The spectral location of the absorption resonance fits well with theoretical calculations of the ISB transition taking the exchange interaction and depolarization shift into account (indicated with black triangles in the figures). When increasing the dopant concentration, the absorption peak broadens significantly. As discussed for GaN/AIN MQWs, the fact that the broadening is more pronounced for the Si-doped samples [see Fig. 5(e)] points to an increase of the interface roughness or the density of structural defects, although no clear evidence of these facts were identified in high-resolution transmission electron microscopy measurements.

\section{FIR absorption in $m$-plane GaN/AIGaN MQWs}

The design of $c$-plane GaN/AlGaN MQWs displaying ISB transitions in the FIR is hindered by the polarization-induced internal electric field, which leads to an increased carrier confinement in the QWs. Thus, nonpolar crystallographic orientations are the most 
promising choice for the development of a GaN-based ISB technology in the FIR range, and we have decided to focus on the $m$ plane to study the effect of Ge-doping. The 40-period $m$ plane $\mathrm{GaN} / \mathrm{Al}_{0.075} \mathrm{Ga}_{0.925} \mathrm{~N}(10 \mathrm{~nm} / 18.5 \mathrm{~nm})$ MQWs presented here are reproduced from a previously-reported Si-doped series [29], designed so that, for low doping densities, two electron levels are clearly confined in the QWs, $\mathrm{e}_{1}$ and $\mathrm{e}_{2}$, separated by $\approx 30 \mathrm{meV}(41 \mu \mathrm{m}$, 7.3 THz), and the third electronic level, $\mathrm{e}_{3}$, is located almost at the conduction band edge of the barriers. In this case, the GaN wells were homogeneously doped with Ge, at concentrations increasing from $5 \times 10^{11} \mathrm{~cm}^{-2}$ to $5 \times 10^{12} \mathrm{~cm}^{-2}$, as described in Table 1. Figures 7(a) and (b) show the schematic description of the samples and the conduction band diagram of one of the QWs in the middle of the stack, indicating the energy of $\mathrm{e}_{1}, \mathrm{e}_{2}$, and $\mathrm{e}_{3}$ and the associated squared wavefunctions. Figure 7(c) shows the HR-XRD $\omega-2 \theta$ scan of the (3-300) reflection of sample S33, containing the most heavily doped MQWs. The thicknesses of the MQW periods for all the samples of the series extracted from the intersatellite distance of such $\omega-2 \theta$ scans are listed in Table 1 , together with the FWHM of the rocking curves for the bulk GaN substrate and the MQW zero-order reflection $\left(0.011 \pm 0.001^{\circ}\right.$ and $0.013 \pm 0.001^{\circ}$, respectively). The values of the FWHM are very similar for the substrate and for the MQW, in agreement with the Si-doped series [29]. In view of these results, we conclude that neither the dopant nature nor the doping density modify the MQW mosaicity, which is rather determined by the substrate.

As an additional evaluation of the structural quality, we recorded AFM images of the samples, as illustrated in Fig. 8(a). The surfaces are characterized by atomic terraces, with a root-mean-square (rms) roughness $<0.3 \mathrm{~nm}$ in $5 \times 5 \mu \mathrm{m}^{2}$ scans. Sample S32 was also characterized by HAADF-STEM, as shown in Figs. 8(b) and (c), where layers with dark and bright contrast correspond to the AlGaN barriers and GaN QWs, respectively. Cross-section images of the stack do not show any extended defects (neither dislocations nor stacking 
faults) over an in-plane length of $\approx 2 \mu \mathrm{m}$. Figure 8 (c) shows two QWs in the middle of the sample viewed along the $<0001>$ zone axis. In the barriers, slight alloy inhomogeneities appear in the form of darker lines parallel to the QW interface, as observed in similar Sidoped structures [25].

The low-temperature (5 K) PL of the nonpolar MQWs is displayed in Fig. 9(a). The spectra consist of a main emission peak at $3.48 \mathrm{eV}$ originating from the MQW and a weaker emission about $100 \mathrm{meV}$ higher in energy that is assigned to the $\mathrm{Al}_{0.075} \mathrm{Ga}_{0.925} \mathrm{~N}$ cap layer. The effect of the doping density on the emission energy and broadening is negligible in the range under study. This is due to the fact that, first, in nonpolar QWs there is no electric field to be screened by carriers, and second, the surface dopant densities described in Table 1 should not have a large effect on the band-to-band transition (the shift due to band filling is $<60 \mathrm{meV}$, and it is partially compensated by bandgap renormalization [48]). The analysis of the CL spectral line-scan on the cross-section of the sample, presented in Fig. 10, confirms that the $\mathrm{Al}_{0.075} \mathrm{Ga}_{0.925} \mathrm{~N}$ emission originates from the cap layer and not from the barriers, where the generated carriers are fully transferred to the QWs. The emission from the QWs is homogeneous in linewidth and intensity all along the structure, as it was also the case for Sidoped QWs with the same structure and similar dopant density [29].

The ISB properties of the structures were assessed by FTIR, and compared to the data obtained in ref. [29] from Si-doped MQWs. In Fig. 9(b), we show the normalized absorption spectra for TM-polarized light from the three Ge-doped samples (peak energies are summarized in Table 1), and the normalized broadening of both the Si-doped and the Gedoped series is compared in Fig. 9(c). The absorption spectra do not vary significantly with increasing Ge doping levels. This behavior is unexpected since in sample S32 the Fermi level should already reach the second electron subband, as confirmed by the saturation of the ISB absorption per pas in Table 1, which should also lead to enhanced scattering. For 
equivalent doping densities, the Ge-doped structures show ISB absorption peaks with slightly smaller broadening than that of the Si-doped structures. When increasing the Gedoping density, we observe a slight widening of the ISB absorption peak and no shift of the ISB energy.

\section{Discussion and conclusions}

In summary, the structural and optical properties of Ge-doped GaN/AlGaN MQWs designed to display ISB absorption in the SWIR, MIR and FIR ranges have been characterized and compared with similar structures doped with Si. For this purpose, we have grown 25-period c-plane GaN/AlN $(1.8 \mathrm{~nm} / 3 \mathrm{~nm})$ MQWs designed to show ISB absorption at $1.70 \mu \mathrm{m}$ $(0.729 \mathrm{eV}), 30$-period $c$-plane $\mathrm{GaN} / \mathrm{Al}_{0.33} \mathrm{Ga}_{0.67} \mathrm{~N}(4 \mathrm{~nm} / 3 \mathrm{~nm}) \mathrm{MQWs}$ designed to display ISB absorption at $5.2 \mu \mathrm{m} \quad(240 \mathrm{meV})$, and 40 -period $m$-plane $\mathrm{GaN} / \mathrm{Al}_{0.075} \mathrm{Ga}_{0.925} \mathrm{~N}$ $(10 \mathrm{~nm} / 18.5 \mathrm{~nm}) \mathrm{MQWs}$ designed to show ISB absorption at $41 \mu \mathrm{m}(30 \mathrm{meV})$.

The results presented above confirm the feasibility of using Ge instead of Si in ISB optoelectronic devices consisting of $\mathrm{GaN} / \mathrm{AlN}$ or GaN/AlGaN heterostructures. However, we can debate on the relevance of using one or the other dopant. For this comparison, it must be kept in mind that $\mathrm{Si}$ and Ge have approximately the same activation energy in GaN [49], and none of them perturbs the growth kinetics of GaN when using PAMBE $[35,40]$. However, Si is known to introduce a strong local deformation of the GaN lattice [50] as well as an enhancement of the interface roughness in GaN/AlGaN QWs [31]. Looking at our results, Si-doped and Ge-doped GaN/AlGaN MQWs are structurally similar both in $c$-plane and $m$ plane crystallographic orientations, with the morphology and mosaicity being determined rather by the substrate and not by the dopant nature or density (in the range under consideration). Only in strongly lattice-mismatched MQWs (GaN/AlN), which exhibits a clear structural degradation with respect to the substrate, Ge results in a systematic structural 
improvement.

If we turn to the optical properties, the evolution of the band-to-band behavior as a function of doping reflects the screening of the internal electric field by free carriers, which is independent of the nature of the dopant. The ISB processes, on the other hand, are more sensitive to parameters like the roughness of the heterointerfaces. Here, results for low doping levels are comparable for MQWs doped with Si or Ge. However, for high doping levels, there is a systematic improvement when using Ge as a dopant, which manifests in narrower absorption bands independent of the spectral region, and thus for different QW size, barrier composition and crystallographic orientations.

\section{Acknowledgements}

The authors acknowledge technical support from Y. Curé and Y. Genuist. Thanks are due to N. Mollard for sample preparation by focused ion beam at the NanoCharacterization Platform (PFNC) in CEA-Minatec Grenoble. The free-standing semi-insulating $m$-GaN substrates were kindly supplied by Suzhou Nanowin Science and Technology Co. Ltd (Nanowin). This work is supported by the EU ERC-StG “TeraGaN" (\#278428) project. A.A. acknowledges financial support by the French National Research Agency via the GaNEX program (ANR-11-LABX-0014). 


\section{References}

[1] Hofstetter D, Baumann E, Giorgetta F R, Théron R, Wu H, Schaff W J, Dawlaty J, George P A, Eastman L F, Rana F, Kandaswamy P K, Guillot F and Monroy E 2010 Intersubband Transition-Based Processes and Devices in AlN/GaN-Based Heterostructures Proc. IEEE 98 1234-48

[2] Beeler M, Trichas E and Monroy E 2013 III-nitride semiconductors for intersubband optoelectronics: a review Semicond. Sci. Technol. 28074022

[3] Suzuki N and Iizuka N 1997 Feasibility Study on Ultrafast Nonlinear Optical Properties of $1.55^{-\mu \mathrm{m}}$ Intersubband Transition in AlGaN/GaN Quantum Wells Jpn. J. Appl. Phys. 36 L1006-8

[4] Iizuka N, Yoshida H, Managaki N, Shimizu T, Hassanet S, Cumtornkittikul C, Sugiyama M and Nakano Y 2009 Integration of GaN/AlN all-optical switch with SiN/AlN waveguide utilizing spot-size conversion Opt. Express 17 23247-53

[5] Vardi A, Sakr S, Mangeney J, Kandaswamy P K, Monroy E, Tchernycheva M, Schacham S E, Julien F H and Bahir G 2011 Femto-second electron transit time characterization in GaN/AlGaN quantum cascade detector at 1.5 micron Appl. Phys. Lett. 99202111

[6] Bellotti E, Driscoll K, Moustakas T D and Paiella R 2009 Monte Carlo simulation of terahertz quantum cascade laser structures based on wide-bandgap semiconductors $J$. Appl. Phys. 105113103

[7] Sun G, Khurgin J B and Tsai D P 2013 Spoof plasmon waveguide enabled ultrathin room temperature $\mathrm{THz} \mathrm{GaN}$ quantum cascade laser: a feasibility study Opt. Express 2128054

[8] Tchernycheva M, Nevou L, Doyennette L, Julien F, Warde E, Guillot F, Monroy E, Bellet-Amalric E, Remmele T and Albrecht M 2006 Systematic experimental and theoretical investigation of intersubband absorption in GaNAlN quantum wells Phys. Rev. B 73125347

[9] Iizuka N, Kaneko K and Suzuki N 2002 Near-infrared intersubband absorption in GaN/AlN quantum wells grown by molecular beam epitaxy Appl. Phys. Lett. 81 18035

[10] Kishino K, Kikuchi A, Kanazawa H and Tachibana T 2002 Intersubband transition in $(\mathrm{GaN})_{\mathrm{m}} /(\mathrm{AlN})_{\mathrm{n}}$ superlattices in the wavelength range from 1.08 to $1.61 \mu \mathrm{m}$ Appl. Phys. Lett. 81 1234-6

[11] Berland K, Stattin M, Farivar R, Sultan D M S, Hyldgaard P, Larsson A, Wang S M and Andersson T G 2010 Temperature stability of intersubband transitions in AlN/GaN quantum wells Appl. Phys. Lett. 97043507

[12] Bayram C, Péré-laperne N and Razeghi M 2009 Effects of well width and growth temperature on optical and structural characteristics of AlN/GaN superlattices grown by metal-organic chemical vapor deposition Appl. Phys. Lett. 95201906

[13] Bayram C 2012 High-quality AlGaN/GaN superlattices for near- and mid-infrared intersubband transitions J. Appl. Phys. 111013514 
[14] Chen G, Li Z L, Wang X Q, Huang C C, Rong X, Sang L W, Xu F J, Tang N, Qin Z X, Sumiya M, Chen Y H, Ge W K and Shen B 2013 Effect of polarization on intersubband transition in AlGaN/GaN multiple quantum wells Appl. Phys. Lett. 102192109

[15] Edmunds C, Tang L, Li D, Cervantes M, Gardner G, Paskova T, Manfra M J and Malis O 2012 Near-Infrared Absorption in Lattice-Matched AlInN/GaN and Strained AlGaN/GaN Heterostructures Grown by MBE on Low-Defect GaN Substrates $J$. Electron. Mater. 41 881-6

[16] Kandaswamy P K, Machhadani H, Bougerol C, Sakr S, Tchernycheva M, Julien F H and Monroy E 2009 Midinfrared intersubband absorption in GaN/AlGaN superlattices on Si(111) templates Appl. Phys. Lett. 95141911

[17] Péré-Laperne N, Bayram C, Nguyen-The L, McClintock R and Razeghi M 2009 Tunability of intersubband absorption from 4.5 to $5.3 \mu \mathrm{m}$ in a GaN/Al ${ }_{0.2} \mathrm{Ga}_{0.8} \mathrm{~N}$ superlattices grown by metalorganic chemical vapor deposition Appl. Phys. Lett. 95 131109

[18] Tian W, Yan W Y, Hui X, Li S L, Ding Y Y, Li Y, Tian Y, Dai J N, Fang Y Y, Wu Z H, Yu C H and Chen C Q 2012 Tunability of intersubband transition wavelength in the atmospheric window in $\mathrm{AlGaN} / \mathrm{GaN}$ multi-quantum wells grown on different $\mathrm{AlGaN}$ templates by metalorganic chemical vapor deposition J. Appl. Phys. 112063526

[19] Machhadani H, Kotsar Y, Sakr S, Tchernycheva M, Colombelli R, Mangeney J, BelletAmalric E, Sarigiannidou E, Monroy E and Julien F H 2010 Terahertz intersubband absorption in GaN/AlGaN step quantum wells Appl. Phys. Lett. 97191101

[20] Sudradjat F F, Zhang W, Woodward J, Durmaz H, Moustakas T D and Paiella R 2012 Far-infrared intersubband photodetectors based on double-step III-nitride quantum wells Appl. Phys. Lett. 100241113

[21] Beeler M, Bougerol C, Bellet-Amalric E and Monroy E 2013 Terahertz absorbing AlGaN/GaN multi-quantum-wells: Demonstration of a robust 4-layer design Appl. Phys. Lett. 103091108

[22] Beeler M, Bougerol C, Bellet-Amalric E and Monroy E 2014 Pseudo-square AlGaN/GaN quantum wells for terahertz absorption Appl. Phys. Lett. 105131106

[23] Edmunds C, Shao J, Shirazi-HD M, Manfra M J and Malis O 2014 Terahertz intersubband absorption in non-polar m-plane AlGaN/GaN quantum wells Appl. Phys. Lett. 105021109

[24] Lim C B, Beeler M, Ajay A, Lähnemann J, Bellet-Amalric E, Bougerol C and Monroy E 2015 Intersubband transitions in nonpolar $\mathrm{GaN} / \mathrm{Al}(\mathrm{Ga}) \mathrm{N}$ heterostructures in the short and mid-wavelength infrared regions JAppl Phys 118014309

[25] Lim C B, Ajay A, Bougerol C, Haas B, Schörmann J, Beeler M, Lähnemann J, Eickhoff M and Monroy E 2015 Nonpolar $m$-plane GaN/AlGaN heterostructures with intersubband transitions in the 5-10 THz band Nanotechnology 26435201

[26] Helman A, Tchernycheva M, Lusson A, Warde E, Julien F H, Moumanis K, Fishman G, Monroy E, Daudin B, Le Si Dang D, Bellet-Amalric E and Jalabert D 2003 Intersubband spectroscopy of doped and undoped GaN/AlN quantum wells grown by molecular-beam epitaxy Appl. Phys. Lett. 83 5196-8 
[27] Kandaswamy P K, Machhadani H, Kotsar Y, Sakr S, Das A, Tchernycheva M, Rapenne L, Sarigiannidou E, Julien F H and Monroy E 2010 Effect of doping on the mid-infrared intersubband absorption in GaN/AlGaN superlattices grown on $\mathrm{Si}(111)$ templates Appl. Phys. Lett. 96141903

[28] Kotani T, Arita M and Arakawa Y 2015 Doping dependent blue shift and linewidth broadening of intersubband absorption in non-polar m-plane AlGaN/GaN multiple quantum wells Appl. Phys. Lett. 107112107

[29] Lim C B, Ajay A, Bougerol C, Lähnemann J, Donatini F, Schörmann J, Bellet-Amalric E, Browne D A, Jiménez-Rodríguez M and Monroy E 2016 Effect of doping on the farinfrared intersubband transitions in nonpolar $m$-plane GaN/AlGaN heterostructures Nanotechnology 27145201

[30] Fritze S, Dadgar A, Witte H, Bügler M, Rohrbeck A, Bläsing J, Hoffmann A and Krost A 2012 High Si and Ge n-type doping of GaN doping - Limits and impact on stress Appl. Phys. Lett. 100122104

[31] Edmunds C, Tang L, Shao J, Li D, Cervantes M, Gardner G, Zakharov D N, Manfra M $\mathrm{J}$ and Malis O 2012 Improvement of near-infrared absorption linewidth in AlGaN/GaN superlattices by optimization of delta-doping location Appl. Phys. Lett. 101102104

[32] Dadgar A, Bläsing J, Diez A and Krost A 2011 Crack-Free, Highly Conducting GaN Layers on Si Substrates by Ge Doping Appl. Phys. Express 4011001

[33] Nenstiel C, Bügler M, Callsen G, Nippert F, Kure T, Fritze S, Dadgar A, Witte H, Bläsing J, Krost A and Hoffmann A 2015 Germanium - the superior dopant in n-type GaN Phys. Status Solidi RRL - Rapid Res. Lett. 9 716-21

[34] Young N G, Farrell R M, Iza M, Nakamura S, DenBaars S P, Weisbuch C and Speck J S 2016 Germanium doping of $\mathrm{GaN}$ by metalorganic chemical vapor deposition for polarization screening applications J. Cryst. Growth 455 105-10

[35] Ajay A, Schörmann J, Jiménez-Rodriguez M, Lim C B, Walther F, Rohnke M, Mouton I, Amichi L, Bougerol C, Den Hertog M I, Eickhoff M and Monroy E 2016 Ge doping of GaN beyond the Mott transition J. Phys. D:Appl. Phys. 49445301

[36] Birner S, Zibold T, Andlauer T, Kubis T, Sabathil M, Trellakis A and Vogl P 2007 nextnano: General Purpose 3-D Simulations IEEE Trans. Electron Devices 54 2137-42

[37] Kandaswamy P K, Guillot F, Bellet-Amalric E, Monroy E, Nevou L, Tchernycheva M, Michon A, Julien F H, Baumann E, Giorgetta F R, Hofstetter D, Remmele T, Albrecht M, Birner S and Dang L S 2008 GaN/AlN short-period superlattices for intersubband optoelectronics: A systematic study of their epitaxial growth, design, and performance J. Appl. Phys. 104093501

[38] Sarigiannidou E, Monroy E, Gogneau N, Radtke G, Bayle-Guillemaud P, BelletAmalric E, Daudin B and Rouvière J L 2006 Comparison of the structural quality in Ga-face and N-face polarity GaN/AlN multiple-quantum-well structures Semicond. Sci. Technol. 21 612-8

[39] Lim C B, Ajay A and Monroy E 2017 Gallium kinetics on m-plane GaN Appl. Phys. Lett. 111022101

[40] Monroy E, Andreev T, Holliger P, Bellet-Amalric E, Shibata T, Tanaka M and Daudin 
B 2004 Modification of GaN(0001) growth kinetics by Mg doping Appl. Phys. Lett. 84 2554

[41] Shao J, Zakharov D N, Edmunds C, Malis O and Manfra M J 2013 Homogeneous AlGaN/GaN superlattices grown on free-standing (1-100) GaN substrates by plasmaassisted molecular beam epitaxy Appl. Phys. Lett. 103232103

[42] Lim C B, Ajay A, Bougerol C, Bellet-Amalric E, Schörmann J, Beeler M and Monroy E 2017 Effect of $\mathrm{Al}$ incorporation in nonpolar m-plane GaN/AlGaN multi-quantum`wells using plasma-assisted molecular-beam epitaxy: Al incorporation in nonpolar m-plane GaN/AlGaN multi-QWs Phys. Status Solidi A 2141600849

[43] Kandaswamy P K, Bougerol C, Jalabert D, Ruterana P and Monroy E 2009 Strain relaxation in short-period polar GaN/AlN superlattices J. Appl. Phys. 106013526

[44] Riyopoulos S 2009 Electrostatically Shielded Quantum Confined Stark Effect Inside Polar Nanostructures Nanoscale Res. Lett. 4 993-1003

[45] Moss T S 1954 Theory of the Spectral Distribution of Recombination Radiation from InSb Proc. Phys. Soc. B 67775

[46] Ajay A, Lim C B, Browne D A, Polaczyński J, Bellet-Amalric E, Bleuse J, den Hertog M I and Monroy E 2017 Effect of doping on the intersubband absorption in $\mathrm{Si}^{-}$and $\mathrm{Ge}^{-}$ doped GaN/AlN heterostructures Nanotechnology 28405204

[47] Kotsar Y, Doisneau B, Bellet-Amalric E, Das A, Sarigiannidou E and Monroy E 2011 Strain relaxation in GaN/AlxGa1-xN superlattices grown by plasma-assisted molecular-beam epitaxy J.Appl. Phys. 110033501

[48] Kleinman D A and Miller R C 1985 Band-gap renormalization in semiconductor quantum wells containing carriers Phys. Rev. B 32 2266-72

[49] Wang H and Chen A-B 2000 Calculation of shallow donor levels in GaN J. Appl. Phys. 877859

[50] Romano L T, Van de Walle C G, Ager J W, Götz W and Kern R S 2000 Effect of Si doping on strain, cracking, and microstructure in GaN thin films grown by metalorganic chemical vapor deposition J. Appl. Phys. 877745 
Tables

Table 1. Structural and optical characteristics of the GaN/AlGaN MQWs under study: dopant nature; surface dopant density; thickness of the MQW period extracted from HRXRD; FWHM of the $\omega$-scan of the (0002) and (3300) x-ray reflections for the $c$-plane and $m$-plane samples, respectively, of the MQWs and of the AIN or GaN substrate; peak energy of the MQW PL emission at $T=5 \mathrm{~K}$; peak energy of the ISB absorption; FWHM of the ISB absorption peak; ISB absorption per pass.

\begin{tabular}{|c|c|c|c|c|c|c|c|c|c|}
\hline Sample & $\begin{array}{l}\text { Dopant } \\
\text { nature }\end{array}$ & $\begin{array}{c}\text { Doping } \\
\text { concentration } \\
\left(\times 10^{12} \mathrm{~cm}^{-2}\right)\end{array}$ & $\begin{array}{l}\text { Period } \\
\text { thickness } \\
(\mathrm{nm})\end{array}$ & $\begin{array}{c}\omega \text {-scan } \\
\text { FWHM } \\
\text { MQW }\left(^{\circ}\right)\end{array}$ & $\begin{array}{c}\text { w-scan } \\
\text { FWHM } \\
\text { substrate } \\
\left({ }^{\circ}\right) \\
\end{array}$ & $\begin{array}{l}\text { PL peak } \\
\text { position } \\
(\mathrm{eV})\end{array}$ & $\begin{array}{c}\text { ISB } \\
\text { absorption } \\
\text { energy } \\
(\mathrm{meV}) \\
\end{array}$ & $\begin{array}{c}\text { ISB } \\
\text { absorption } \\
\text { FWHM } \\
(\mathrm{meV}) \\
\end{array}$ & $\begin{array}{c}\text { ISB } \\
\text { absorption } \\
\text { per pass }(\%)\end{array}$ \\
\hline S11 & $\mathrm{Si}$ & 6 & $4.8 \pm 0.1$ & 0.199 & 0.056 & 3.37 & 710 & 85 & 2.1 \\
\hline $\mathrm{S} 12$ & $\mathrm{Ge}$ & 6 & $4.8 \pm 0.1$ & 0.167 & 0.046 & 3.35 & 720 & 94 & 1.8 \\
\hline S13 & $\mathrm{Si}$ & 20 & $4.8 \pm 0.1$ & 0.180 & 0.061 & 3.45 & 830 & 108 & 8.9 \\
\hline S14 & $\mathrm{Ge}$ & 20 & $4.8 \pm 0.1$ & 0.154 & 0.058 & 3.46 & 760 & 90 & 6.6 \\
\hline S15 & $\mathrm{Si}$ & 60 & $4.4 \pm 0.1$ & 0.176 & 0.057 & 3.51 & 860 & 189 & 9.5 \\
\hline S16 & $\mathrm{Ge}$ & 60 & $4.3 \pm 0.1$ & 0.149 & 0.063 & 3.63 & 820 & 110 & 8.6 \\
\hline $\mathrm{S} 21$ & $\mathrm{Si}$ & 2 & $7.0 \pm 0.1$ & 0.190 & 0.212 & 3.41 & 249 & 45 & 4.7 \\
\hline S22 & $\mathrm{Ge}$ & 2 & $7.0 \pm 0.1$ & 0.188 & 0.209 & 3.41 & 272 & 59 & 4.1 \\
\hline S23 & $\mathrm{Si}$ & 10 & $7.0 \pm 0.1$ & 0.187 & 0.210 & 3.42 & 286 & 80 & 5.8 \\
\hline S24 & $\mathrm{Ge}$ & 10 & $7.0 \pm 0.1$ & 0.185 & 0.210 & 3.41 & 276 & 77 & 7.4 \\
\hline S25 & $\mathrm{Si}$ & 20 & $6.9 \pm 0.1$ & 0.189 & 0.206 & 3.43 & 280 & 140 & 15 \\
\hline S26 & $\mathrm{Ge}$ & 20 & $6.9 \pm 0.1$ & 0.187 & 0.204 & 3.49 & 273 & 90 & 16 \\
\hline S31 & $\mathrm{Ge}$ & 0.5 & $27.5 \pm 0.1$ & 0.014 & 0.012 & 3.48 & 25 & 12 & 19 \\
\hline S32 & $\mathrm{Ge}$ & 2 & $28.2 \pm 0.1$ & 0.013 & 0.010 & 3.48 & 24 & 13 & 19 \\
\hline S33 & $\mathrm{Ge}$ & 5 & $29.3 \pm 0.1$ & 0.013 & 0.011 & 3.48 & 24 & 15 & 13 \\
\hline
\end{tabular}




\section{Figure captions}

Figure 1. (a) Schematic of the GaN/AIN MQW structures. (b) Band diagram of one of the QWs and location of the first $\left(\mathrm{e}_{1}\right)$ and second $\left(\mathrm{e}_{2}\right)$ electron levels obtained using a selfconsistent 8-band k.p Schrödinger-Poisson solver. (c) HR-XRD $\omega-2 \theta$ scan of the (0002) reflection of Si- and Ge-doped heterostructures (S15 and S16, respectively). Experimental data are compared to a simulation which assumes that the MQWs present the in-plane lattice parameters of an AlGaN layer with the average Al concentration of the MQW.

Figure 2. Low temperature $(T=5 \mathrm{~K})$ PL intensity of the (a) Ge-doped and (b) Si-doped GaN/AlN MQWs. The spectra are normalized by their maximum value and vertically shifted for clarity. Black triangles mark the transition energies calculated using a self-consistent 8band k.p Schrödinger-Poisson solver that takes into account the screening of the internal electric field by free carriers. Normalized TM-polarized ISB absorption of the (c) Ge-doped and (d) Si-doped MQWs measured by FTIR spectroscopy. The spectra are normalized by their maximum value and vertically shifted for clarity. Black triangles mark the transition energies calculated using a self-consistent 8-band k.p Schrödinger-Poisson solver and corrected to account for the exchange interaction and depolarization shift. (e) Normalized broadening $(\triangle \mathrm{E} / \mathrm{E})$ of the ISB absorption peak as a function of the doping density $\left(N_{\mathrm{S}}\right)$.

Figure 3. CL spectral line-scan on the cross-section of sample S16 measured at $T=10 \mathrm{~K}$. The intensity is color-coded on a logarithmic scale. The sketch on the right side shows the corresponding sequence of layers.

Figure 4. (a) Schematic of the $\mathrm{GaN} / \mathrm{Al}_{0.33} \mathrm{Ga}_{0.67} \mathrm{~N}$ MQW structures. (b) Band diagram of one of the QWs and location of the first $\left(\mathrm{e}_{1}\right)$ and second $\left(\mathrm{e}_{2}\right)$ electron levels obtained using a selfconsistent 8-band k.p Schrödinger-Poisson solver. (c) HR-XRD $\omega-2 \theta$ scan of the (0002) 
reflection of Si- and Ge-doped heterostructures (S25 and S26, respectively). Experimental data are compared to a simulation which assumes that the MQWs present the in-plane lattice parameters of an AlGaN layer with the average $\mathrm{Al}$ concentration of the MQW. The simulation does not take into account the buffer layer of the GaN-on-Si(111) template. This buffer contains a periodic GaN/AlGaN heterostructure (period $\approx 20 \mathrm{~nm}$ ).

Figure 5. Normalized PL intensity of the (a) Ge-doped and (b) Si-doped GaN/Al $1_{0.33} \mathrm{Ga}_{0.67} \mathrm{~N}$ MQWs measured at $T=5 \mathrm{~K}$. The spectra are normalized by their maximum value and vertically shifted for clarity. Black triangles mark the transition energies calculated using a self-consistent 8-band k.p Schrödinger-Poisson solver that takes into account the screening of the internal electric field by free carriers. Normalized TM-polarized ISB absorption of the (c) Ge-doped and (d) Si-doped MQWs measured by FTIR spectroscopy. The spectra are normalized by their maximum value and vertically shifted for clarity. Black triangles mark the transition energies calculated using a self-consistent 8-band k.p Schrödinger-Poisson solver and corrected to account for the exchange interaction and depolarization shift. (e) Normalized broadening $(\Delta \mathrm{E} / \mathrm{E})$ of the ISB absorption peak as a function of the doping density $\left(N_{\mathrm{S}}\right)$.

Figure 6. CL spectral line-scan on the cross-section of sample S26 measured at $T=10 \mathrm{~K}$. The intensity is color-coded on a logarithmic scale. The sketch on the right side shows the corresponding sequence of layers.

Figure 7. (a) Schematic of the $m$-plane $\mathrm{GaN} / \mathrm{Al}_{0.075} \mathrm{Ga}_{0.925} \mathrm{~N}$ MQW structures. (b) Band diagram of one of the QWs and location of the first $\left(e_{1}\right)$, second $\left(e_{2}\right)$ and third $\left(e_{3}\right)$ electron levels obtained using a self-consistent 8-band k.p Schrödinger-Poisson solver. (c) HR-XRD $\omega-2 \theta$ scan of the (3-300) reflection of S33. Experimental data are compared to a simulation which assumes that the MQWs are fully strained on GaN. 
Figure 8. (a) AFM image $\left(5 \times 5 \mu \mathrm{m}^{2}\right)$ of sample $\mathrm{S} 31$ showing an rms surface roughness of $0.28 \mathrm{~nm}$. (b)-(c) Cross-section HAADF-STEM images of sample S32 viewed along $<0001>$. Layers with dark and bright contrast correspond to the $\mathrm{AlGaN}$ barriers and $\mathrm{GaN}$ QWs, respectively.

Figure 9. (a) Low temperature $(T=5 \mathrm{~K})$ PL intensity of the Ge-doped $m$-plane MQWs. The spectra are normalized by their maximum value and vertically shifted for clarity. (b) Normalized absorption for TM-polarized light measured at $5 \mathrm{~K}$ by FTIR spectroscopy. (c) Normalized broadening $(\Delta \mathrm{E} / \mathrm{E})$ of the ISB absorption peak as a function of the doping density $\left(N_{\mathrm{S}}\right)$. The triangular data points correspond to the Ge-doped MQWs under study, whereas the square data points correspond to similar Si-doped MQWs from ref. [29].

Figure 10. CL spectral line-scan on the cross-section of sample S33 measured at $T=10 \mathrm{~K}$. The intensity is color-coded on a logarithmic scale. The sketch on the right side shows the corresponding sequence of layers. 
Figure 1

(a)

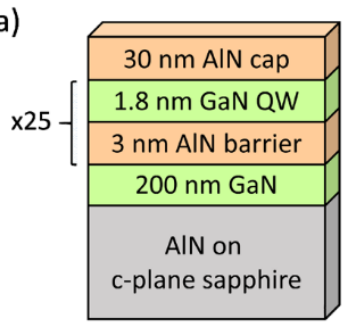

(b)

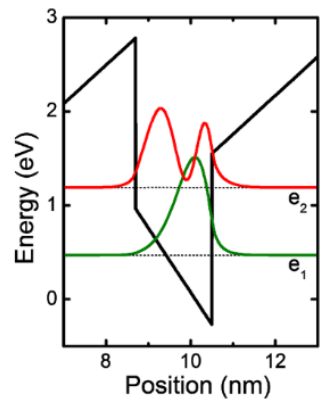

(c)

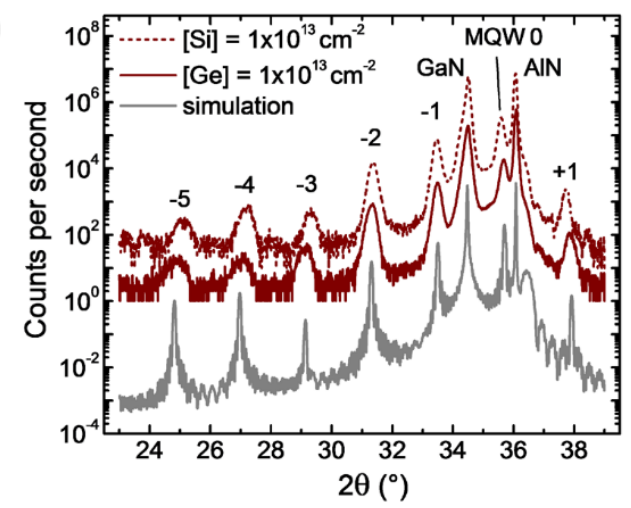


Figure 2
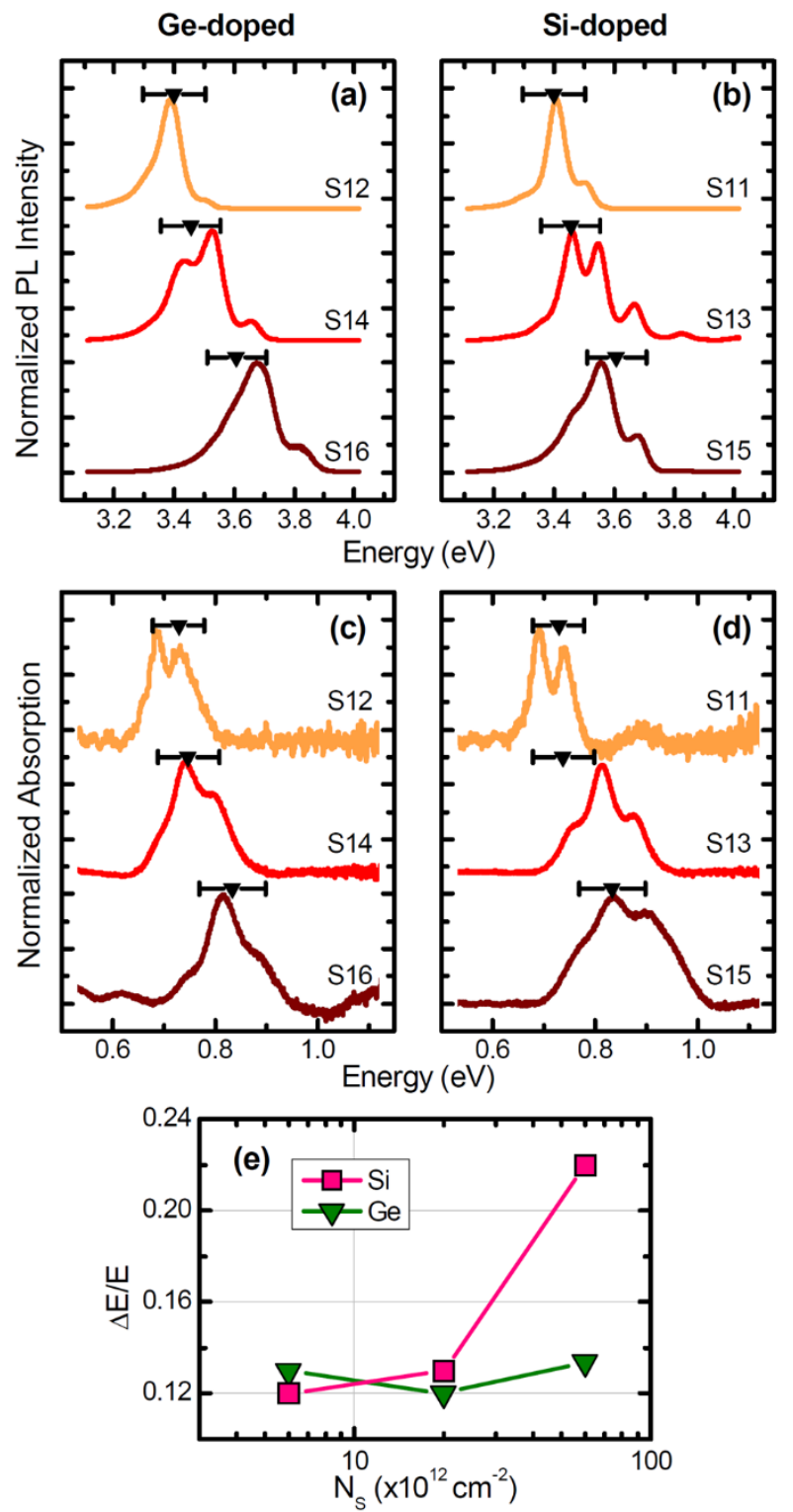


\section{Figure 3}

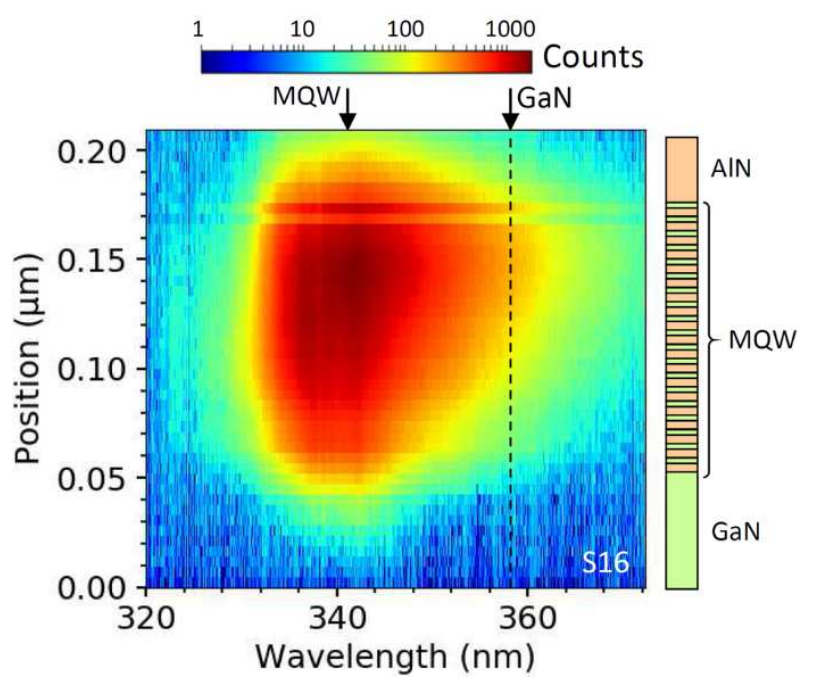

Figure 4
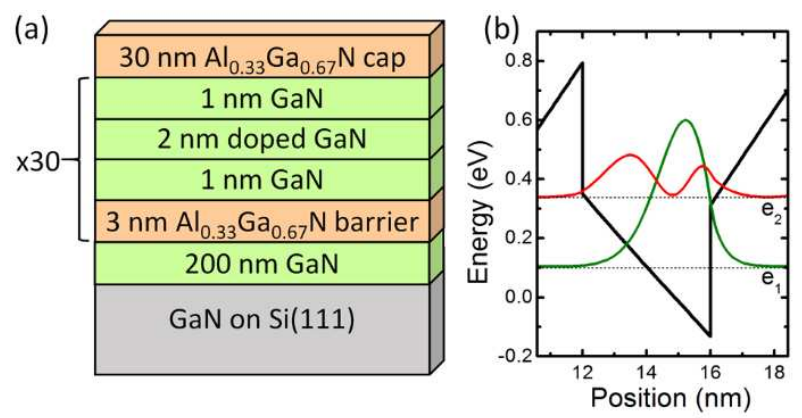

(c)

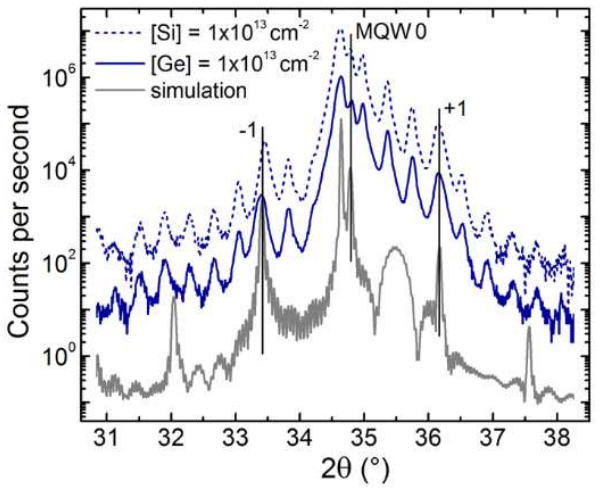


Figure 5
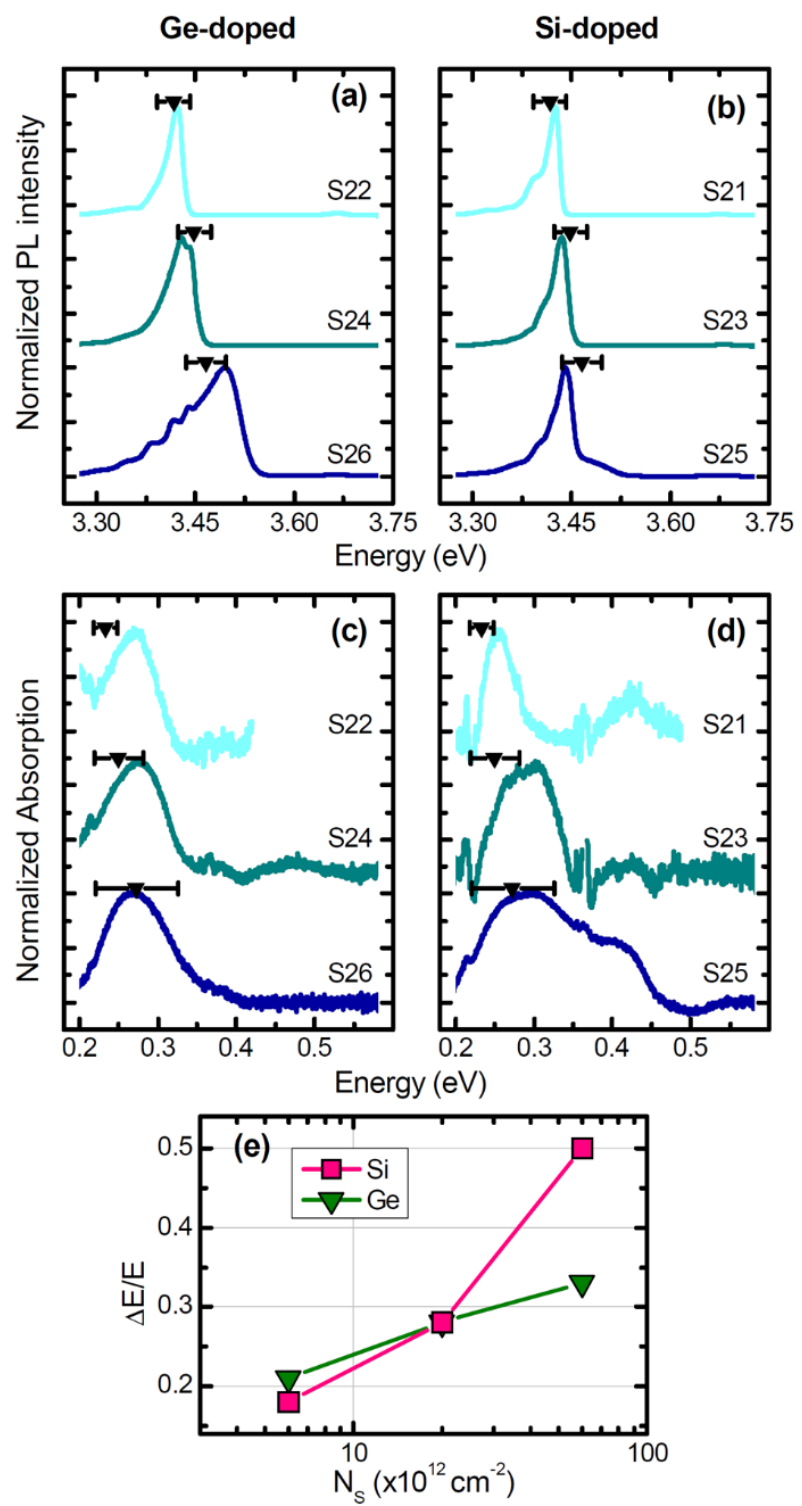
Figure 6

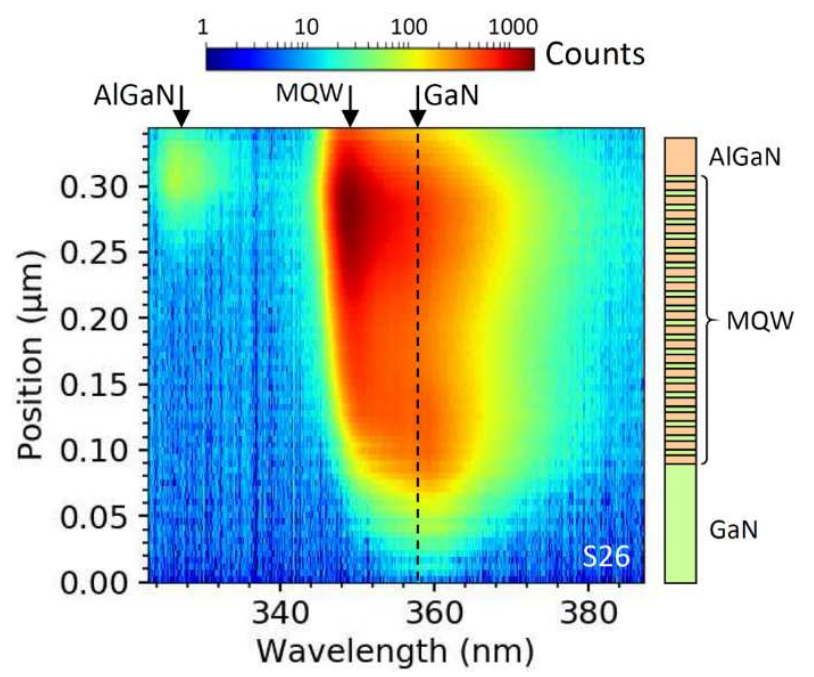

Figure 7

(a)

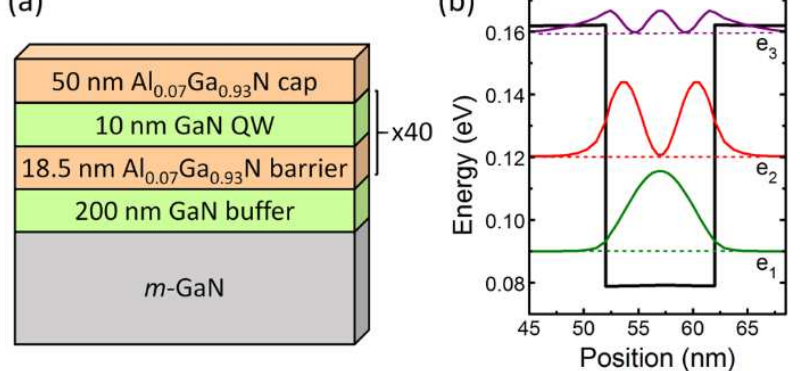

(c)

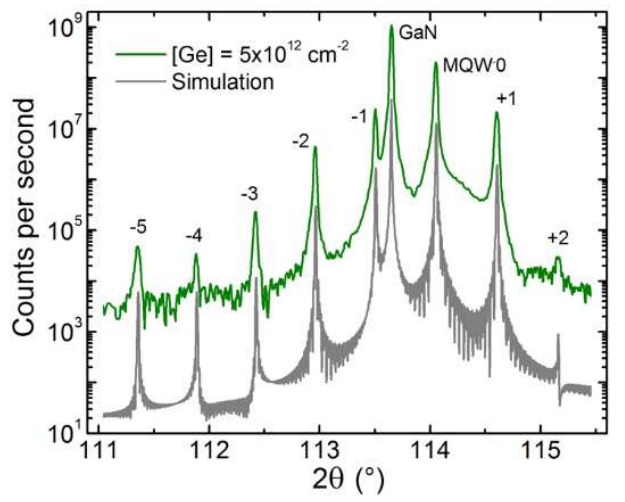


Figure 8
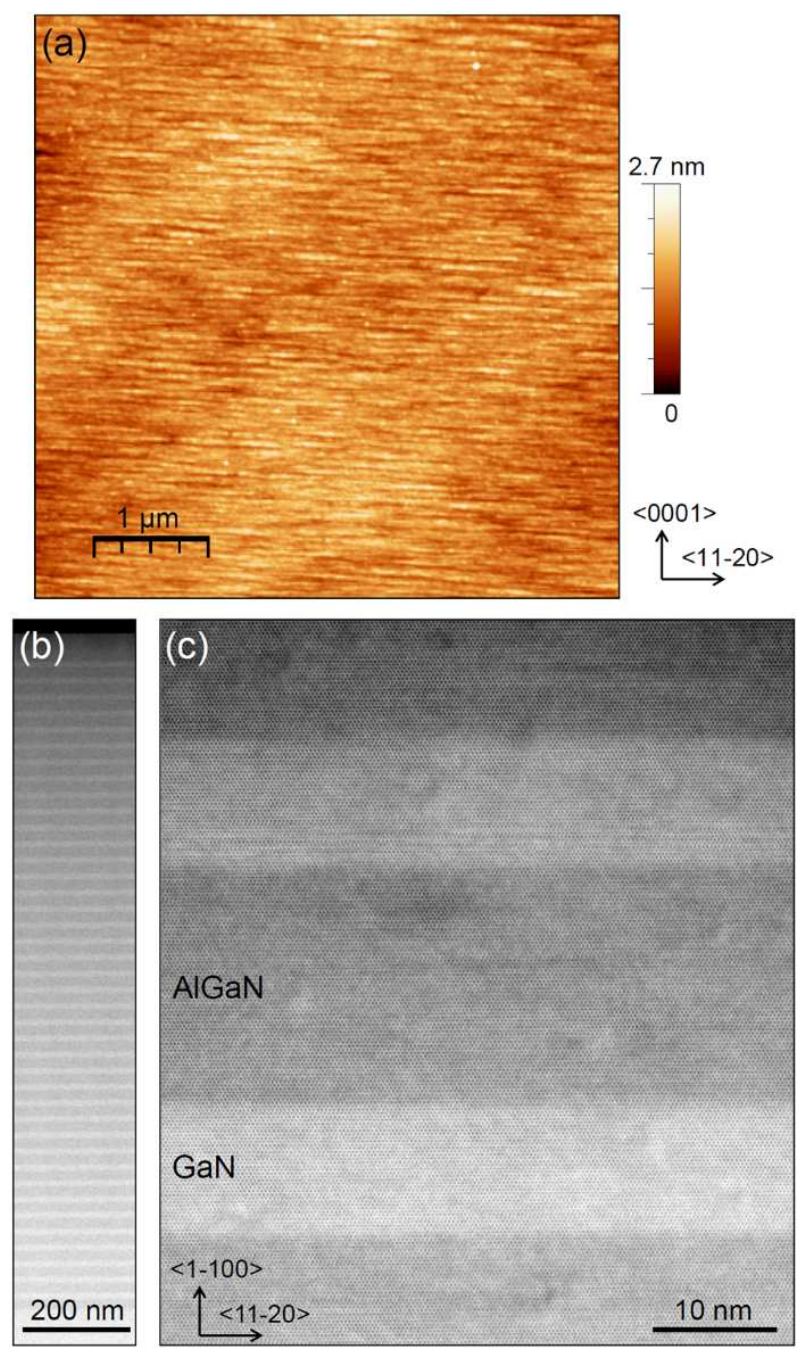
Figure 9
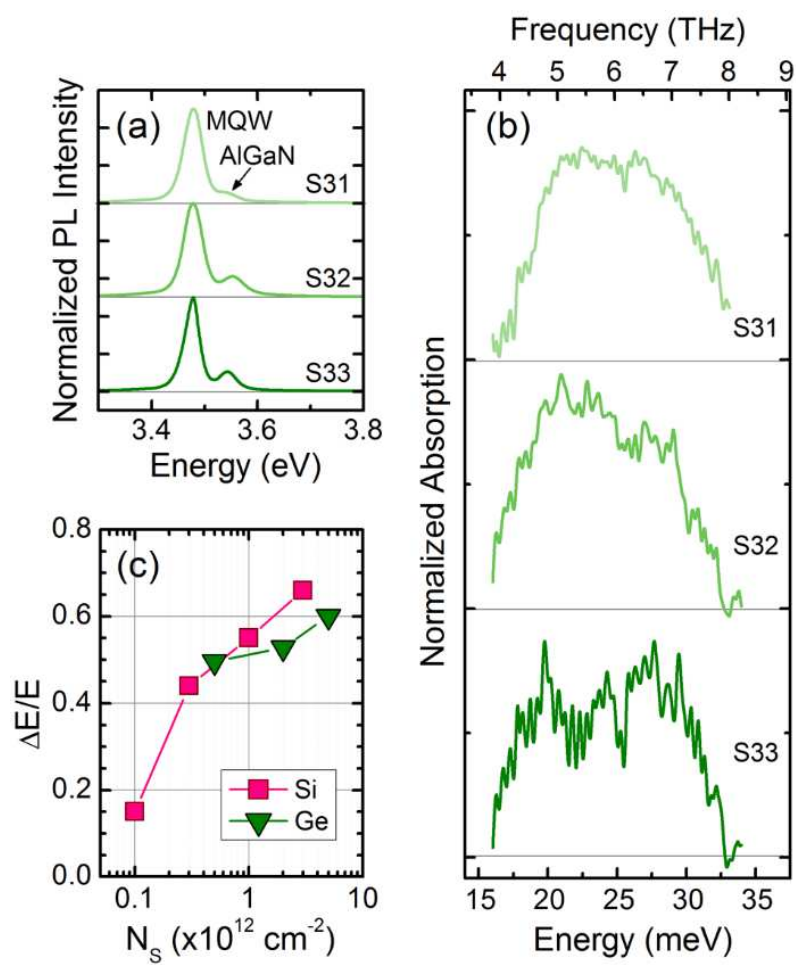

Figure 10

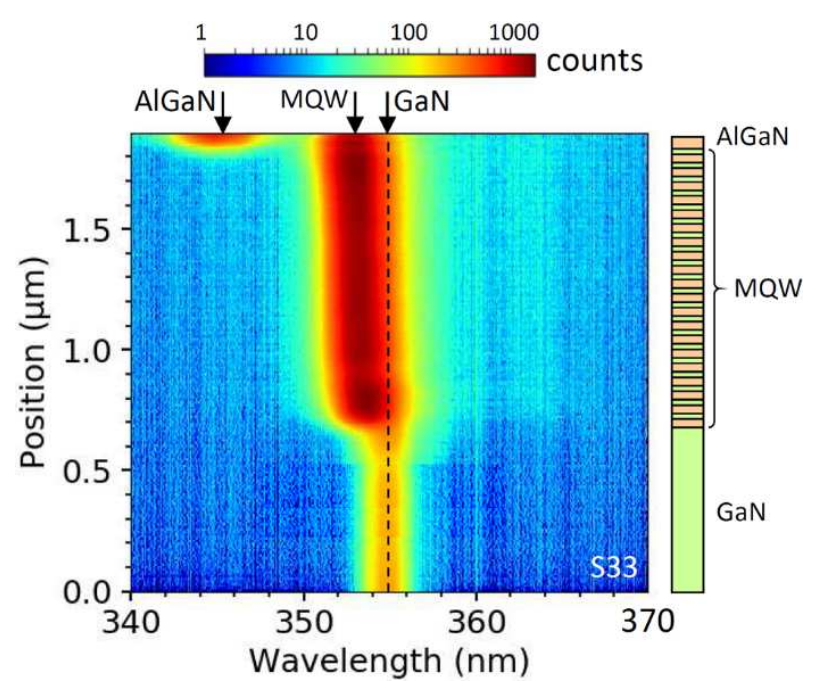

